\title{
Investigating SI Skills: Methodological and didactic reflections
}

\begin{abstract}
The paper presents and discusses an empirical study of simultaneous interpreting (SI) between English and Danish, carried out with a view to improving the SI teaching situation. Since the study is still in its preliminary stages, the paper focusses on methodological and didactic reflec-tions.

One important hypothesis is that the main difference between SI and non-literary translation (hereafter referred to as "translation") is to be found in transmission conditions and process rather than in objective. The paper gives the rationale of this hypothesis and, by means of examples from the empirical data, tries to examine its truth-value.

For methodological and diagnostic reasons, the study concentrates on the rendition of progressive "meaning" in English and Danish. The paper explains how the subjects' mastery of progressive aspect, which is realized in very different ways in the two lan- guages, may be used as one indicator for the communicative adequacy of their target texts ${ }^{1}$ (hereafter referred to as TTs).
\end{abstract}

\section{Introduction}

The overall objective of the study is to understand and improve SI teaching and to contribute to existing theory in the field. A more specific objective is to determine to which degree teachers and learners of interpreting may benefit from skills acquired in previous translation classes. To achieve this goal, I describe and analyze SI performances at different levels of competence (beginners and professionals) and compare them with translations at comparable levels.

The empirical data comprise four groups of subjects: interpreters and translators at various levels of competence.

Group I: The starting point and focus of the analysis is the level of simultaneous interpreting reached by 9 cand.ling.merc. (CLM) ${ }^{2}$ students

1 I do not distinguish between written texts and oral discourse. To me, both interpreters and translators receive and produce "texts". For a discussion of the concept of "text", see for instance Enkvist (1989).

2 The CLM programme is a Master's programme in translation and interpreting in one language, open to students holding a BA degree in LSP. The programme mainly provides translator-relevant subjects, but it also includes an introductory course in interpreting (consecutive as well as simultaneous). 
of English in their final and fifth year at the Aarhus School of Business. The subjects in this first group are regarded as beginners in the field of simultaneous interpreting.

Group II: The CLM performances are then compared with those of 7 interpreter-trainees near the end of a six-month postgraduate course at the Centre for Conference Interpreting at the Copenhagen Business School. It is assumed that these are near-professionals ${ }^{3}$.

Groups III and IV: The performances in the interpreter-groups are then compared with those of translators at comparable levels. The third and principal translator-group consists of 13 CLM students who have just (ie one or two years previously) entered the master's programme. These subjects are regarded as near-beginners in the field of translation. The fourth group, whose role is principally that of control, consists of two professional, practising translators (translatorer).

The source texts (hereafter referred to as STs) - one Danish and one En-glish - were political, topical ${ }^{4}$ speeches given orally (though on the basis of carefully planned manuscripts) to the interpreter-subjects to interpret in January and February 1991. The translator-subjects then translated a written version of the same texts in March 1991.

In order to reduce the number of variables in the investigation and as I do not consider the use of dictionaries to be an intrinsic quality of the translation process ${ }^{5}$, the translator-subjects were asked not to consult any sources of information other than their own memory and background knowledge. Thus, by depriving all subjects of access to external knowledge, it was thought that their different tasks could be more easily defined and analyzed.

\section{Hypotheses}

In my investigation I operate with three interlinked hypotheses: The first and principal hypothesis deals with similarites and differences between SI and translation. The second deals with the communicative adequacy

3 As the enrolment in this course requires the passing of an entrance test for "interpreting ability", the subjects in this fourth group are regarded as particularly talented. This, together with their status as interpreter-trainees, justifies the epithet of near-professional.

4 The topicality of the STs is important for the third hypothesis, dealing with progressive aspect. It was assumed that a speaker's expressing "progressive" viewpoints (see 2.3.1.) of a real on-going situation would encourage "progressive" renditions.

5 This of course does not mean to say that dictionaries and reference books are not very important aids in the work of any translator. 
of the TTs produced by simultaneous interpreters and translators. The third hypothesis, which is mainly diagnostic and consequently subordinate to the other two, deals with subjects' mastery of progressive aspect.

\subsection{First Hypothesis}

The first and principal hypothesis 6 is that the main difference between SI and translation is to be found in transmission conditions and process rather than in objective. If this hypothesis is not true, the interpreter-subjects and the translator-subjects in my investigation should produce significantly different types of TTs, as they would thus adhere to different concepts of objective. If this is true, they should produce rather similar TTs. Furthermore, if this hypothesis is not true, translator-students (my groups I and III) should not have to learn interpreting and interpretingtrainees (my group II) would be ill-advised to acquire translation training before contemplating full-time interpreting careers. However, if this hypothesis is true, it would mean that a person's practice of translation should enhance his/her skills in interpreting and vice versa.

This first hypothesis is assumed to be in opposition to conventional wisdom 7 . The activities of interpreting and translating are often regarded as so different - in process as well as in objective - that it is generally argued that the learning of translation even constitutes a counterproductive factor in interpreting training. As a result of this, many teachers choose to introduce their students to interpreting by lecturing on how the objective is the transfer of a sender's "intentions, ideas, etc.", not the "translation" of his/her words. If my first hypothesis holds good, such a lecture on objective belongs in the previous translation classes or should take the form of a reminder of already acquired knowledge.

\subsubsection{Communicative Objective}

Taking my point of departure in some of the literature on interpreting as well as on translation, I shall now attempt to show why I think the two

6 This hypothesis is greatly inspired by a study carried out by Dollerup, Fluger, and Zoëga in Copenhagen. See for instance Dollerup, Fluger, and Zoëga (1992).

7 It is, of course, always dangerous to venture such a definition of the "general state of affairs". It can never be proved and is therefore bound to be deemed an exaggeration. However, the point to note is not whether the postulate is true or false, but the fact that this subjective feeling of mine (when thinking of my own education and training) was one of my reasons for formulating this first and principal hypothesis. 
tasks share the same communicative objective. Firstly, most writers seem to agree that "sense" should be emphasized as the guiding principle. Seleskovitch (1978:335) argues that in both translation and interpreting "sense is made of the act of speech, and sense remains the guiding principle”. Lörscher (1989:49) defines translation in a similar way:

.. sense (as the content of a text) and denotation (as the reference to extralinguistic facts) are bound to an individual language. The task of translation must therefore be to convey the same denotation and the same sense into another language by means of the meaning of that language.

Another similarity between the two tasks is that the metaphor of a "communicative triangle" is frequently used to describe the situation of communication via a mediator. As regards translation, Neubert (1985:7), for instance, describes the task as "language mediation", and Pergnier (1978:203) states:

Translation is the extreme case of this general situation [of communication between "encoder" and "decoder"], owing to the fact that the message is not only received by the receiver, but also re-emitted in an entirely different "code".

As regards interpreting, the same "triangle" appears. Kirchhoff (1976), for instance, puts forward the three-party two-language model (see also Kondo 1990:61) to describe the situation: (1) sender, who, using language A, wants to convey some sort of message to the receiver(s), whose identity may be known or unknown; (2) language mediator, who transfers this message to the receiver(s); and (3) receiver(s), who, using language B, want(s) to receive the sender's message. We may therefore conclude that both interpreting and translation are tripartite, interlingual tasks, performed with a communicative purpose.

\subsubsection{Transmission Conditions}

Those factors which influence the transmission process are: time, medium, and mode ${ }^{8}$. In the following I shall try to explain how SI normally differs from translation.

Time: First of all, the interpreter works under a lot of time pressure. As s/he operates concurrently with the sender, the process cannot be interrupted and the ST has to be perceived, analyzed and transferred in segments. This means a heavy burden on the interpreter's short-time

8 See also Dollerup (1978:19). 
memory capacity (depending on the EVS 9 ), just like s/he must be able to share his/her attention between the STs and TTs ${ }^{10}$. Furthermore, this simultaneity makes him/her exclusively dependent on his/her own (internal) knowledge.

Medium: Obviously, both STs and TTs are spoken. This may have both advantages and disadvantages. The basic advantage is that all parties are present in the communicative context, which may make up for less-than-perfect utterances by the interpreter as well as the speaker. The major disadvantage of the oral medium is of course that the interpreter cannot regress in the ST and is equally prevented from editing and revising the TT.

Mode: In principle, both STs and TTs are to be characterized by oral language. However, even if this is usually true for the interpreter's output (see for instance Kopczynski 1980:16), this is far from always the case with the speaker's output. The fact that the STs at conferences (and in the SI classroom) are often carefully planned, perhaps even read aloud from a written-language manuscript, force interpreters to cope with STs that are very similar in mode to those of the translator. Perhaps it would then be truer to say that the real difference between the two tasks, as far as mode is concerned, lies more in the characteristics of the TTs than in the STs ${ }^{11}$. However, it should also be mentioned that the difference between the two modes might be seen as a continuum rather than a binary system (see for instance Shlesinger 1990).

\subsection{Second Hypothesis}

The second hypothesis is that, in spite of rather strenuous working conditions and an extremely complex process, TTs produced by interpreters may be just as communicatively adequate ${ }^{12}$ as translations of the same

9 EVS (Ear-Voice Span) is the necessary time lag between the speaker's and the interpreter's production of a certain segment. See for instance Barik (1975:290), who suggests that the ability to segment the incoming message at linguistically appropriate places is one of the critical factors in successful SI.

10 Henderson (1982:25) argues that attention-sharing is the only element unique to SI.

11 It may be argued that this written characteristic of many STs is contradictory to the nature of SI. Lederer (1978:324) even suggests that as interpreting is free speech, it should always be investigated in connection with spontaneous speech and not with prepared statements or papers being read out.

12 While working with this part of the investigation, I use this tentative definition: An adequate TT fulfils the communicative objective of the ST. This is greatly inspired by the Skopos theory, presented in Reiss and Vermeer (1984), and for instance discussed and described by Nord (1992) and Pöchhacker (1992). 
texts. If this hypothesis is not true, the TTs produced by one subject category - say, the translator-subjects - should generally be more successful than those produced by the other - say, the interpreter-subjects. However, if this hypothesis is true, the performances in one category should prove to be at least as adequate as those in another. Consequently, possibly due to its oral medium with a more "dynamic" situation, interpreting may in certain circumstances be a better choice of language mediation than the time-consuming act of translation, a point of considerable encouragement for the interpreter-students.

\subsection{Third Hypothesis}

The third and subordinate hypothesis, which is closely linked with the methodological and diagnostic strategy of the investigation, is that a mediator's reception and production of progressive aspect in English may be used as one indicator for the communicative adequacy of his/her TT. If this hypothesis is not true, it is possible for a subject to produce a communicatively adequate ST without the adequate usage of progressive aspect. However if the hypothesis is true, a mediator's failure to convey the sender's "progressive" intentions means that the whole TT is communicatively inadequate. Thus, according to this hypothesis, a communicatively successful TT can only be produced if the subject masters progressive aspect in the two languages.

\subsubsection{Progressive Aspect}

Aspect concerns the manner in which a verbal action is experienced or regarded. By choosing either progressive or simple verb forms (morphological means) in English, the sender can signal whether he sees the action as in progress or completed. In other words, rather than being a description of reality, progressive aspect concerns the sender's view of a situation (see King 1983:131).

\subsubsection{Danish vs. English}

In all but a few cases, the marking of aspect in Danish is optional (Østergaard 1979:95). This means that the obligatory status of the aspect category in English presents a particular problem for the English-speaking Dane (see Davidsen-Nielsen et al 1982:53) in perception as well as in production. The Danish subjects of my investigation are no exception. 
Danish has (at least) two ways of expressing progressive meaning. One is by means of fixed, lexicalized phrases.

EX1: Thomas går og ${ }^{13}$ driller.

[Thomas is teasing]

The other is by means of unmarked, simple verb forms, whose "progressive" meaning is then conveyed by inherent semantic features of the verb or understood by the receiver on the basis of the co-text (eg adverbials) and/or the context (an on-going situation). Consequently, while receiving a sender's "progressive" viewpoint expressed by morphological means in English, the mediator has to render this lexically or by other means in Danish - and vice versa. Here is an example from the Danish ST, in which the "progressive" viewpoint is expressed mainly by verb meanings and context ${ }^{14}$ :

EX2: Medens jerntappet brasede sammen i $\phi$ st, kunne man i alverdens medier se Gorbatjov drikke gravol i vest.

[While the Iron Curtain was collapsing in the East, the world's media showed Mr Gorbachev taking part in its wake in the West]

\section{Results}

In the light of the investigation's three hypotheses, I shall now try to assess some of the results.

\section{1. ad First Hypothesis: SI vs. translation}

Allowing for differences of media (such as speech errors, hesitations, unsemantic pauses, etc), the TTs of the investigation are strikingly similar. In most cases, all subjects seem to have struggled with the same sort of problems, which indicates that their concepts of objective have been equally similar. For instance, when dealing with EX2, interpreters and translators alike had to solve these problems:

Semantics (ie find adequate renditions of individual lexical items)

1. jerntæppe [Iron Curtain]

2. brase sammen [collapse, crumble, die]

3. alverdens medier [the world's media]

4. gravøl [wake; funeral feast; directly translated "grave beer"]

13 This is just one of many such fixed phrases.

14 According to the speaker's viewpoint, the verbs denote temporary, incomplete actions in the past, which at some time or other were simultaneous (see Leech 1987:22), the first action probably framing the second. 


\section{Sender viewpoint}

progressive aspect

As far as the time factor is concerned, it was interesting to discover how relatively similar the two tasks appeared, in the sense that the translator-subjects (both near-beginners and professionals) seem to have chosen a rather "simultaneous" working method, not bothering to regress, revise and edit. However, this discovery should not change the basic assumption about the two tasks: The interpreter-subjects were forced to cope with extreme time pressure and segmentation of ST and TT, whereas the translator-subjects at least could choose to work more at leisure.

\section{2. ad Second Hypothesis: Communicative adequacy}

The interpreting performances are often surprisingly adequate. Here is an example from the group of beginners (group I). Even if it is flawed in some ways - for instance because "crushing down" (2) is non-existent 15 in English and because the lexcial item of "alverdens medier" (3) is not rendered - the receiver is in no doubt as to the speaker's intentions:

EX3: While the Iron Curtain (1) was crushing down (2) in the East, [..] Gorbachev could be seen drinking the toast (4) to this in the west.

The same cannot be said for EX4, which is a translation by one of the near-beginners (group III). Even if all lexical items are rendered, the receiver may still be in doubt as to the intentions of the sender.:

EX4: While the iron curtain (1) fell (2) in the East, you could se Gorbatjov drink funeral beer (4) in the west in the medias all over the world (3).

\section{3. ad Third Hypothesis: Progressive Aspect}

It cannot, as yet, be proved that this third, diagnostic hypothesis is true. Though a lack of progressive mastery in most cases coincide with an otherwise flawed TT (like EX4), it is far from certain that this is always so. There might be at least three reasons for a subject not to render a sender's "progressive" intentions:

1) The subject does not master progressive aspect.

2) The subject masters progressive aspect, but fails to comprehend the sender's "progressive" intentions.

15 Usually "crush" is transitive and not a phrasal verb, or it is intransitive and means "to become crushed". "Crumble" or "tumble down" is probably what the subject had in mind. 
3) The subject masters progressive aspect and succeeds in comprehending the sender's "progressive" intentions, but for some reason (perhaps failing short-term memory, lack of concentration, failing semantic anticipation) fails to articulate this in the TT.

The following is an example of the third reason. The TT is taken from the group of interpreters who are characterized as near-professionals (group II). As the subject's A language (ie mother tongue) is English, it must be assumed that she masters progressive aspect. However, only the second part of the sentence contains a progressive verb form. It is assumed - though one can never be sure - that this is due to the simultaneous process. While rendering the first part of the sentence, the subject fails to anticipate the framing characteristic of the first action:

EX5: As the Iron Curtain (1) fell (2) in eastern Europe, it was possible to see Gorbachev drinking the funeral celebratory drink (4) in the west.

Even if the sender's progressive intentions are not perfectly rendered, this part of the TT is far from communicatively inadequate. The fact that this is possible rather disproves the third hypothesis.

The following, rather interesting, example equally disproves the hypothesis. It is taken from the group of beginner-interpreters (group I). Though the performance is communicatively inadequate, it seems to indicate a certain mastery of progressive aspect.

EX6: While the er, [..] the Iron er Curtain (1) was, was being dismatling, was being dismanted (2), you could see-er Gorbachev drinking funeral beer (4) [ha!'] ${ }^{16}$ at the, at the summit.

\section{Concluding Remarks}

In this article I have outlined the general plan of my investigation, I have explained the rationale of the hypotheses and given examples of a few results in this connection. As far as the first and second hypotheses are concerned, it may be concluded - even at his early stage - that the subjects of the investigation seem to adhere to similar concepts of objective and that the TTs of the interpreter-subjects are generally as communicatively adequate as those of the translator-subjects. However, as far as the third hypothesis is concerned, it must be concluded that the focus on pro-

16 The subject laughs at his own rendition. 
gressive aspect in the analysis is insufficient to describe the communicative adequacy of the TTs. Thus, these results cannot be used for generalizations about the nature of the tasks and the competence of the subjects.

There may be many reasons for the fallacy of the third hypothesis. One important reason is that, as the sender's "progressive" viewpoint may be hard to define, it is also very difficult to determine whether progressive usage in a certain example is obligatory or facultative. Another reason may be (as shown in 3.3) that a subject's failure to render a sender's "progressive" intentions may have many explanations and that it is usually impossible to tell them from each other.

A third perhaps even more important reason is that the focus on one "grammatical" phenomenon - no matter how differently it is realized in the two languages and no matter how the sender's usage of it influences the message - is too limited. As the success of a TT depends on the receiver's impression as a whole, the assessment of of a mediator's performance should never be based on segment analysis alone. Therefore, it may be concluded that when assessing the TTs, in order to prove or disprove the first and second hypotheses, I need other and more relevant, if less manageable, parametres.

\section{References}

Barik, Henri Charles (1975): Simultaneous Interpretation: Qualitative and Linguistic Data. In: Language and Speech Vol.18, pp. 272-294.

Davidsen-Nielsen, Niels \& Færch, Claus \& Harder, Peter (1982): The Danish Learner. Kent: Antony Taylor.

Dollerup, Cay (1978): Omkring sproglig transmission, University of Copenhagen, Department of English. In: Anglica et Americana 3.

Dollerup, Cay \& Fluger, Lena \& Zoëga, Anne (1992): Interpreting and Translation: Two sides of the same coin, Århus. In: Hermes No.8, pp. 43-53.

Dollerup, Cay \& Loddegaard, Anne (eds.) (1992): Teaching Translation and Interpreting: Training, Talent and Experience. Amsterdam: John Benjamins Publishing Company.

Enkvist, Nils Erik (1989): From Text to Interpretability: A contribution to the discussion of basic terms in text linguistics. In: Heydrich, Wolfgang \& Neubauer \& Peöfi \& Sözer, pp. 369-382.

Fruchtman, Maya \& Kaddari, Menahem Zevi (eds.) (1990): Stylistics and the Literary Text: A special issue, Bar-Ilan University Press. In: Hebrew Linguistics Issue (Balsanut 'ivrit) No. 28-29-30.

Gerver, David \& Sinaiko, H. Wallace (eds.) (1978): Language Interpretation and Communication. New York: Plenum Press. 
Henderson, John (1982): Some Psychological Aspects of Simultaneous Interpreting,. In: Fremdsprache Vol.1, pp. 21-27.

Heydrich, Wolfgang \& Neubauer, Fritz \& Petöfi, János S. \& Sözer, Emel (eds.) (1989): Connexity and Coherence. Berlin: Walter de Gruyter.

King, Larry D. (1983): The Semantics of Tense, Orientation, and Aspect in English. In: Lingua Vol.59, pp. 101-154.

Kirchhoff, Hella (1976): Das dreigliedrige, zweisprachige Kommunikationssystem Dolmetschen. In: Le Langage et l'Homme Vol.31, pp. 21-27.

Kondo, Masaomi (1990): What Conference Interpreters Should not be Expected to Do. In: The Interpreters' Newsletter No.3, pp. 59-65.

Kopczynski, Andrzej (1980): Conference Interpreting: Some linguistic and communicative problems: Dissertation presented to the Institute of Applied Linguistics. Poznan: Warsaw University.

Lederer, Marianne (1978): Simultaneous Interpretation - Units of Meaning and other Features. In: Gerver \& Sinaiko, pp. 323-332.

Leech, Geoffrey N. (1987): Meaning and the English Verb.London: Longman.

Lörscher, Wolfgang (1989): Models of the Translation Process: Claim and reality. In: Target Vol.1, No.1, pp. 43-68.

Neubert, A. (1985): Text and Translation, VEB Verlag Enzyklopädie Leipzi In: Übersetzungswissenschaftliche Beiträge 8.

Nord, Christiane (1992): Text Analysis in Translator Training. In: Dollerup \& Loddegaard, pp. 39-48.

Pergnier, Maurice (1978): Language-Meaning and Message-Meaning: Towards a Sociolinguistic Approach to Translation. In: Gerver \& Sinaiko, pp. 199-204.

Pettersson, Thore (ed.) (1979): Aspectology: Workshop at the Fifth Scandinavian Conference of Linguistics, Frostvallen April 27-29 1979, Almqvist \& Wiksell International.

Pöchhacker, Franz (1992): The Role of Theory in Simultaneous Interpreting. In: Dollerup \& Loddegaard, pp. 211-220.

Reiss, Katharina \& Vermeer, Hans J. (1984): Grundlegung einer allgemeinen Translationstheorie. Tübingen: Niemeyer.

Seleskovitch, Danica (1978): Interpreting for International Conferences, Pen and Booth, Washington D.C., (Translation and adaptation of Seleskovitch, D. (1968), Paris).

Shlesinger, Miriam (1990): Factors Affecting the Applicability of the Oral-literate Continuum to Interpretation Research. In: Fructman \& Kaddari, Hebrew Linguistics No.28-29-30, pp. 49-56.

Østergaard, Frede (1979): The Progressive Aspect in Danish. In: Pettersson, pp. 89-109. 
